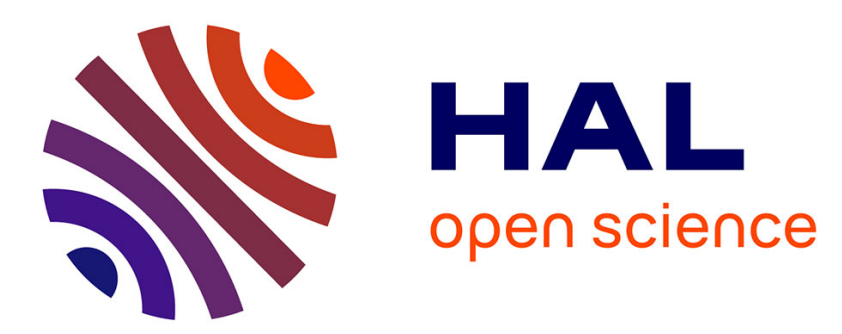

\title{
Numerical simulation of unsteady cavitation in liquid hydrogen flows
}

\author{
Eric Goncalves da Silva, Dia Zeidan
}

\section{To cite this version:}

Eric Goncalves da Silva, Dia Zeidan. Numerical simulation of unsteady cavitation in liquid hydrogen flows. International Journal of Engineering Systems Modelling and Simulation, 2017, 9 (1), pp.41. 10.1504/IJESMS.2017.10002571 . hal-01457192v2

\section{HAL Id: hal-01457192 \\ https://hal.science/hal-01457192v2}

Submitted on 3 Mar 2017

HAL is a multi-disciplinary open access archive for the deposit and dissemination of scientific research documents, whether they are published or not. The documents may come from teaching and research institutions in France or abroad, or from public or private research centers.
L'archive ouverte pluridisciplinaire HAL, est destinée au dépôt et à la diffusion de documents scientifiques de niveau recherche, publiés ou non, émanant des établissements d'enseignement et de recherche français ou étrangers, des laboratoires publics ou privés. 
Numerical Simulation of Unsteady Cavitation in Liquid Hydrogen Flows

\title{
Eric Goncalvès
}

Ensma - Pprime, UPR 3346 CNRS, Poitiers, France

E-mail: eric.goncalves@ensma.fr

\section{Dia Zeidan*}

School of Basic Sciences and Humanities

German Jordanian University, Amman, Jordan

E-mail: dia.zeidan@gju.edu.jo

*Corresponding author

\begin{abstract}
An unsteady cavitation model in liquid hydrogen flow is studied in the context of compressible, two-phase, one-fluid inviscid solver. This is accomplished by applying three conservation laws for mixture mass, mixture momentum and total energy along with gas volume fraction transport equation, with thermodynamic effects. Various mass transfers between phases are utilized to study the process under consideration. A numerical procedure is presented for the simulation of cavitation due to rarefaction and shock waves. Attention is focused on cavitation in which the simulated fluid is liquid hydrogen in cryogenic conditions. Numerical results are in close agreement with theoretical solutions for several test cases. The current numerical results show that liquid hydrogen flow can be accurately modeled using an accurate inviscid approach to describe the features of thermodynamic effects on cavitation.
\end{abstract}

Keywords: Two-phase flow; heat and mass transfer; liquid and hydrogen, cavitation; homogeneous model; splitting techniques, inviscid simulation.

\section{Reference $\cdots$}

Biographical notes: Eric Goncalvès is a Professor in the Aeronautical Engineering School ISAE-ENSMA, Poitiers, France. Currently, he is the head of the Department Fluid Mechanics and Aerodynamics. His research interests are related to the modelling and the simulation of flows for which the density is variable such as compressible flow, two-phase flow and cavitation. Recent work include shock wave boundary layer interaction, thermal effects in cavitation and investigation of three-dimensional effects on cavitation pocket.

Dia Zeidan is currently a Tenure-track Assistant Professor in the School of Basic Sciences and Humanities at the German Jordanian University, Amman, Jordan. His expertise is in the mathematical modelling and numerical simulations of multiphase fluid flow problems. Recent work also includes hyperbolicity and conservativity resolution related to two-phase flows equations in the context of the Riemann problem and simulations of such flows over a wide range of non-equilibrium behaviours.

\section{Introduction}

Thermodynamics of thermosensitive fluids is an essential phenomenon of cavitation dynamics arising in cryogenic fluids. These fluids are typically characterized by variations in fluid properties such as the temperature, with special intense to vapour pressure. In cryogenic fluids, however, the density ratio of liquid-vapour is lower than the density of typical fluids such as cold water which leads to vaporization of liquid mass to sustain a cavity. The evaporative cooling effects are more evident where the temperature of the liquid phase near the liquidvapour interface is depressed below the free-stream temperature. Although the temperature depression is negligible in cold water, it remains a continuing interest in cavitation. Indeed, for cavitation, the local cooling effects delay such phenomenon and decrease the fluid local vapour pressure that leads to a lower cavity pressure. Consequently, this implies that it is useful for cryogenic pumps to establish a relatively improved performance.

Earlier research on thermal effects was generally focused on achieving correlations for temperature depression as a function of flow conditions and liquid properties. This has included the B-factor theory that characterize the sensitivity of fluids to thermodynamic effects (Stahl 
et al. 1956, Moore \& Ruggeri 1968). In Hsiao \& Chahine (2002), Hsiao et al. (2006), the authors used the Rayleigh-Plesset equation to investigate such thermodynamics. In this model, the governing equations are composed by three balance equations for the mixture quantities coupled with a macroscopic model for the bubble dynamics based on the Rayleigh-Plesset equation. This model is capable of handling either single bubbles or clouds of bubbles that grow and decrease through a pressure field (Fujikawa et al. 1980, Rodio et al. 2012). In the case where heat transfer is negligible, the phase change is driven by inertia effects. Yet, when thermal effects are involved, the liquid inertia become rapidly negligible and the evolution is controlled by the heat flux provided by the liquid at the bubble surface (Florschuetz \& Chao 1965, Prosperetti \& Plesset 1978). The widely used modeling approach in cavitation is based on averaged two-phase flow models of the one-fluid formulation. However, there are different approaches within these models according to the assumptions made on the local thermodynamic equilibrium along with the slip conditions between phases. In general, the seven-equation models are regarded as the most complete models due to their non-equilibrium process between phases (Ambroso et al. 2012, Baer \& Nunziato 1986, Chalons et al. 2011). Further, these models have been investigated and applied to metastable states and evaporation front dynamics (Saurel \& Metayer 2001, Ishimoto \& Kamijo 2004). Another set of similar models, the five-equation model, is also derived on the basis velocity and pressure equilibrium (Allaire et al. 2002, Kapila et al. 2001, Kreeft \& Koren 2010). This also can be expressed as a four-equation model by assuming thermal equilibrium between phases. With such formulation, the authors in (Utturkar et al. 2005, Tseng \& Shyy 2010, Huang et al. 2014) adapted a set of models to simulate turbulent cavitating flows in cold water within cryogenic applications. These models, however, utilizes three conservation laws for mixture quantities (mass, momentum, energy) along with a mass equation for the vapour or liquid density including a cavitation source (Utturkar et al. 2005). Yet, this family of models is not thermodynamically well-posed and does not account for the main thermodynamic constraints features (Goncalves \& Patella 2011, Zeidan et al. 2007). The authors in (Downar-Zapolski et al. 1996, Schmidt et al. 2010) have applied the Homogeneous Relaxation Model to boiling flow processes taking into account the mass fraction equation with a relaxation term which is estimated from experimental data.

The objective of this paper is to investigate cavitating flows in liquid hydrogen within cryogenic conditions. This is based on a transport-equation for the void fraction in which the mass transfer between phases appear explicitly and closed by assuming its proportionality with the velocity divergence. The vapour pressure is assumed to vary linearly with the temperature though the parameter $d P_{v a p} / d T$. Validations have shown the ability of such models to correctly simulate cavitation pockets in both water and freon R-114 (Goncalves 2013, Goncalves \& Charriere 2014, Goncalves 2014, Charriere et al. 2015, Goncalves \& Zeidan 2015). In order to investigate thermal effects in $\mathrm{LH}_{2}$ cryogenic cavitating flows, one-dimensional cavitation tube problems are proposed. Such rarefaction tube problems involving cavitation are one of the most used case to study the behaviour of phase transition models and to test and develop numerical schemes (Saurel \& Metayer 2001, Barberon \& Helluy 2005, Saurel et al. 2008, Zein et al. 2010, Causon \& Mingham 2013, Spina et al. 2014, Pelanti \& Shyue 2014).

This paper is organized as follows. In Section 2, we present the governing equations, the mass transfer closure relations and the mixture equation of states. Section 3 describes the finite volume scheme adopted and the integration of the source term. The numerical results and the influence of parameters are presented in Section 4.

\section{Equations and models}

The homogeneous mixture approach is used to model the two phase flows of interest in the current paper. Further, the two phases are assumed to be sufficiently well mixed with very small particles of the dispersed phase and the two phase move at equal velocities and share the same pressure $P$. Although the temperature-equalizing time is larger than the pressure and velocity relaxation times, as noted in (Kapila et al. 2001), it is possible to consider a single-temperature model as an approximate model of flow if the difference of phase temperatures is not too big.

\subsection{A four-equation single-temperature model}

The model consists in three conservation laws for mixture quantities and an additional equation for the void ratio (Goncalves 2013). We present below the inviscid one-dimensional equations, expressed with the vector of variables $w=(\rho, \rho u, \rho E, \alpha)$ :

$$
\begin{aligned}
\frac{\partial \rho}{\partial t}+\frac{\partial \rho u}{\partial x} & =0 \\
\frac{\partial(\rho u)}{\partial t}+\frac{\partial\left(\rho u^{2}+P\right)}{\partial x} & =0 \\
\frac{\partial(\rho E)}{\partial t}+\frac{\partial(\rho u H)}{\partial x} & =0 \\
\frac{\partial \alpha}{\partial t}+u \frac{\partial \alpha}{\partial x} & =K \frac{\partial u}{\partial x}+\frac{\dot{m}}{\rho_{I}} \\
K=\frac{\rho_{l} c_{l}^{2}-\rho_{v} c_{v}^{2}}{\frac{\rho_{l} c_{l}^{2}}{1-\alpha}+\frac{\rho_{v} c_{v}^{2}}{\alpha}} & \text { and } \frac{1}{\rho_{I}}=\frac{\frac{c_{v}^{2}}{\alpha}+\frac{c_{l}^{2}}{1-\alpha}}{\frac{\rho_{l} c_{l}^{2}}{1-\alpha}+\frac{\rho_{v} c_{v}^{2}}{\alpha}} .
\end{aligned}
$$

In the above, $E=e+u^{2} / 2$ and $H=h+u^{2} / 2$ denote the mixture total energy and the mixture total enthalpy, respectively. $\rho_{I}$ is the interfacial density, $\dot{m}$ is the mass transfer between phases and $c_{k}$ the speed of sound of the phase $k$. 


\subsubsection{Pure phase EOS}

The liquid density $\rho_{l}$ is assumed to be in its equilibrium state at the reference temperature: $\rho_{l}=\rho_{l}^{\text {sat }}\left(T_{r e f}\right)$. In addition to that, the vapour density $\rho_{v}$ follows the stiffened gas EOS and varies with the temperature. The convex stiffened gas EOS relations are (see (Metayer et al. 2004)):

$$
\begin{aligned}
P(\rho, e) & =(\gamma-1) \rho(e-q)-\gamma P_{\infty} \\
P(\rho, T) & =\rho(\gamma-1) C_{v} T-P_{\infty} \\
T(\rho, h) & =\frac{h-q}{C_{p}}
\end{aligned}
$$

where $\gamma=C_{p} / C_{v}$ is the heat capacity ratio, $C_{p}$ and $C_{v}$ are thermal capacities, $q$ the energy of formation and $P_{\infty}$ is the constant reference pressure. The speed of sound $c$ is given by:

$$
c^{2}=\gamma \frac{P+P_{\infty}}{\rho}=(\gamma-1) C_{p} T
$$

\subsubsection{Closure relation for the mass transfer}

Assuming the mass transfer is proportional to the divergence of the velocity, it is possible to develop a family of models (Goncalves 2013, Goncalves \& Charriere 2014) in which the mass transfer is expressed as:

$$
\dot{m}=\frac{\rho_{l} \rho_{v}}{\rho_{l}-\rho_{v}}\left(1-\frac{c^{2}}{c_{\text {wallis }}^{2}}\right) \frac{\partial u}{\partial x} .
$$

Here $c_{\text {wallis }}$ is the propagation velocity of acoustic waves without mass transfer (Wallis 1967). This speed of sound is expressed as a weighted harmonic mean of speeds of sound of each phase:

$$
\frac{1}{\rho c_{\text {wallis }}^{2}}=\frac{\alpha}{\rho_{v} c_{v}^{2}}+\frac{1-\alpha}{\rho_{l} c_{l}^{2}} \text {. }
$$

\subsubsection{Mixture EOS}

To close the system and to compute the mixture pressure and the mixture temperature, an equation of state for the mixture is necessary. In the present study, two formulations are compared: a mixture of stiffened gas and a sinus law.

\section{A mixture of stiffened gas EOS}

Assuming pressure equilibrium between phases, an expression for the pressure can be obtained as a function of the void ratio $\alpha$ and the vapour mass fraction $Y$ :

$$
\begin{aligned}
P(\rho, e, \alpha, Y) & =(\gamma(\alpha)-1) \rho(e-q(Y)) \\
& -\gamma(\alpha) P_{\infty}(\alpha), \\
\frac{1}{\gamma(\alpha)-1} & =\frac{\alpha}{\gamma_{v}-1}+\frac{1-\alpha}{\gamma_{l}-1}, \\
q(Y) & =Y q_{v}+(1-Y) q_{l},
\end{aligned}
$$

$$
\begin{aligned}
P_{\infty}(\alpha) & =\frac{\gamma(\alpha)-1}{\gamma(\alpha)}\left[\alpha \frac{\gamma_{v}}{\gamma_{v}-1} P_{\infty}^{v}\right. \\
& \left.+(1-\alpha) \frac{\gamma_{l}}{\gamma_{l}-1} P_{\infty}^{l}\right], \\
Y & =\frac{\alpha \rho_{v}}{\rho} .
\end{aligned}
$$

Further, assuming thermal equilibrium between phases, the mixture temperature is expressed as:

$$
T(\rho, h, Y)=\frac{h_{l}-q_{l}}{C_{p_{l}}}=\frac{h_{v}-q_{v}}{C_{p_{v}}}=\frac{h-q(Y)}{C_{p}(Y)},
$$

where

$$
C_{p}(Y)=Y C_{p_{v}}+(1-Y) C_{p_{l}} .
$$

The speed of sound within the mixture can be represented as a function of the enthalpy of each phase (Goncalves \& Patella 2009):

$$
\rho c^{2}=(\gamma-1)\left[\frac{\rho_{v} \rho_{l}}{\left(\rho_{l}-\rho_{v}\right)}\left(h_{v}-h_{l}\right)\right],
$$

where the enthalpies of pure phase $h_{l}$ and $h_{v}$ are computed with the mixture temperature $T$.

\section{A modified sinusoidal EOS}

A sinusoidal relation can be considered for the current mixture flows (Goncalves \& Patella 2010). When the pressure is smaller than $P_{\text {vap }}(T)+\Delta P$, the following relationship applies:

$$
\begin{aligned}
P(\alpha, T) & =P_{\text {vap }}(T) \\
& +\left(\frac{\rho_{l}^{\text {sat }}-\rho_{v}^{\text {sat }}}{2}\right) c_{\text {sinus }}^{2} \sin ^{-1}(1-2 \alpha) .
\end{aligned}
$$

This EOS introduces a small non-equilibrium effect on the pressure quantified by the quantity $\Delta P$. For a void ratio value of 0.5 , the pressure is equal to the saturation pressure $P_{v a p}(T)$ at the local temperature $T$. This temperature is evaluated using the relation (17). The saturation values $\rho_{l}^{s a t}$ and $\rho_{v}^{\text {sat }}$ are evaluated at the reference temperature $T_{r e f}$. The quantity $c_{\text {sinus }}$, which has the dimension of a velocity, is a parameter of the model. The pressure continuity between the liquid and the mixture is given by:

$$
\begin{aligned}
\frac{\pi}{2} \frac{\rho_{l}^{\text {sat }}-\rho_{v}^{\text {sat }}}{2} c_{\text {sinus }}^{2} & =\rho_{l}^{\text {sat }}\left(\gamma_{l}-1\right) C_{v_{l}} T_{\text {ref }} \\
& -P_{\infty}^{l}-P_{\text {vap }}\left(T_{r e f}\right),
\end{aligned}
$$

which determines $c_{\text {sinus }}$ for given values of saturation conditions. It is worth note that the vaporization pressure varies linearly with the temperature by

$$
P_{v a p}(T)=P_{v a p}\left(T_{r e f}\right)+\frac{d P_{v a p}}{d T}\left(T-T_{r e f}\right),
$$

where the constant quantity $d P_{\text {vap }} / d T$ is evaluated using a thermodynamic table. When $d P_{v a p} / d T=0$, one find 
the isothermal model. Furthermore, the speed of sound in the mixture can be written as (Goncalves \& Patella 2010):

$$
\begin{gathered}
c^{2}=\frac{\frac{\rho_{v} \rho_{l}}{\rho\left(\rho_{l}-\rho_{v}\right)}\left(h_{v}-h_{l}\right) \frac{d P_{v a p}}{d T}+\rho C_{p}(Y) c_{T}^{2}}{\rho C_{p}(Y)-\frac{d P_{v a p}}{d T}}, \\
c_{T}^{2}=\left(\frac{\partial P}{\partial \rho}\right)_{s}=\left(\frac{\partial P}{\partial \rho}\right)_{T}=\frac{c_{\text {sinus }}^{2}}{2 \sqrt{\alpha(1-\alpha)}},
\end{gathered}
$$

where $c_{T}$ is the isothermal speed of sound.

\subsubsection{Hyperbolicity}

Without mass transfer, the four equations form a system of conservation laws having a hyperbolic nature. The eigenvalues of the system are:

$$
\lambda_{1}=u-c_{\text {wallis }}, \quad \lambda_{2,3}=u \quad \text { and } \quad \lambda_{4}=u+c_{\text {wallis }} .
$$

However, when heat and mass transfer occur, the system is still hyperbolic with the following eigenvalues:

$$
\lambda_{1}=u-c, \quad \lambda_{2,3}=u \quad \text { and } \quad \lambda_{4}=u+c,
$$

where $c$ is the mixture speed of sound which depends on the EOS formulation.

\section{Numerics}

The conservation laws governing both models is written as

$$
\frac{\partial w}{\partial t}+\frac{\partial F(w)}{\partial x}=S(w)
$$

where $w$ is the vector of variables, $F$ the convective flux and $S$ the source term. We focus herein on finite volume schemes. Regular meshes are considered, whose size $\Delta x$ is such that: $\Delta x=x_{i+1 / 2}-x_{i-1 / 2}$ with the usual time step $\Delta t$, where $\Delta t=t^{n+1}-t^{n}$. Also, we let $w_{i}^{n}$ be the approximate value of $w\left(x, t^{n}\right)$ in the cell centered on $x_{i}$. A discrete form of the system can be written as:

$$
\Delta x \frac{w_{i}^{n+1}-w_{i}^{n}}{\Delta t}+F_{i+1 / 2}^{n}-F_{i-1 / 2}^{n}=S_{i}^{n} \Delta x,
$$

where $F_{i+1 / 2}$ is the numerical flux through the cell interface $x_{i+1 / 2} \times\left[t^{n}, t^{n+1}\right]$. The time step should comply with CFL (Courant-Freidrichs-Lewy) condition in order to guarantee some stability requirement. Finally, the numerical flux through the cell interface is computed with both the first-order Rusanov scheme (Rusanov 1961) and the second-order Jameson-Schmidt-Turkel scheme (Jameson et al. 1981).

\subsection{Treatment of the source term}

The numerical simulations of the initial-boundary value problems are accomplished using splitting approach. One starts in solving the source-free homogeneous part of the whole system:

$$
\frac{\partial w}{\partial t}+\frac{\partial F(w)}{\partial x}=0 .
$$

This is followed by solving the system of ordinary differential equations describing the mass transfer between phases to obtain the complete solution:

$$
\frac{d w}{d t}=S(w, \nabla w)
$$

\subsection{Inlet and outlet boundary conditions}

The numerical treatment of the boundary conditions is based on the use of the characteristic relations of Euler equations. The number of variables to impose at boundaries is given by the number of positive characteristics. The characteristic relations obtained for the 4-equation system are (Goncalves 2013):

$$
\begin{aligned}
-c^{2}\left(\rho^{c}-\rho^{s}\right)+\left(P^{c}-P^{s}\right) & =0, \\
\left(P^{c}-P^{s}\right)+\rho c\left(V^{c}-V^{s}\right) & =0, \\
\left(P^{c}-P^{s}\right)-\rho c\left(V^{c}-V^{s}\right) & =0, \\
\rho\left(\alpha^{c}-\alpha^{s}\right)-K\left(\rho^{c}-\rho^{s}\right) & =0 .
\end{aligned}
$$

The variables with superscript $c$ denote the variables to be computed at the boundary. Variables with superscript $s$ denote the variables obtained by the current numerical scheme.

At inflow, we impose the initial values of the void ratio, densities of pure phases and the velocity. The pressure is evaluated with the relation (31) and all variables can be evaluated at the boundary.

At outflow, the static pressure is imposed. The variables are computed with three characteristic relations (29), (30) and (32).

\section{Simulation of double rarefaction cases}

These cases are similar to those proposed in (Saurel et al. 2008) with hot water. It consists in a one meter long tube filled with a liquid and a weak volume fraction of vapour $\alpha=0.01$ is added. An initial discontinuity of velocity $u_{0}$ is set at $0.5 \mathrm{~m}$, the left velocity is $-u_{0}$ and the right velocity is $u_{0}$. The stretch of the liquid leads to the creation of a cavitation area in the middle of the tube (see Figure 1). Without mass transfer, the solution involves two expansion waves. As gas is present, the pressure cannot become negative. To maintain positive pressure, the gas volume fraction increases due to the gas mechanical expansion and creates a pocket. Liquid water is expanded until the saturation pressure is reached then evaporation appears and quite small amount of vapour is created. The solution with phase transition is composed of four expansion waves. The extra two expansion waves correspond to the evaporation fronts (see Figure 2). The evaporation creates a cooling effect and a temperature 
Table 1 Parameters of the stiffened gas EOS for $L H_{2}$ at $T=22.1 \mathrm{~K}$

\begin{tabular}{|c|c|c|c|c|c|}
\hline & $\gamma$ & $P_{\infty}(\mathrm{Pa})$ & $q(\mathrm{~J} / \mathrm{kg})$ & $C_{p}(\mathrm{~J} / \mathrm{K} . \mathrm{kg})$ & $\rho_{\text {sat }}\left(\mathrm{kg} / \mathrm{m}^{3}\right)$ \\
\hline liquid & 2.8 & 2. $10^{5}$ & $-2.2110^{5}$ & 10875 & 68.78 \\
\hline vapour & 1.38 & 0 & $1.6610^{5}$ & 13090 & 2.50 \\
\hline
\end{tabular}

depression is observed inside the cavity. According to the initial velocity $u_{0}$, the cooling effect can be strong.

A simple heat balance between the two phases can estimate the scale of temperature difference $\Delta T^{*}$ caused by thermal effects.

$$
\Delta T^{*}=\frac{\rho_{v} L_{v a p}}{\rho_{l} C_{p_{l}}}
$$

where $L_{v a p}$ is the latent heat.

The $B$-factor is estimated as the ratio between the actual temperature drop and $\Delta T^{*}$. By assuming that the whole liquid contributes to the heat necessary for the vapor, we obtain (Franc \& Michel 2004):

$$
B=\frac{\Delta T}{\Delta T^{*}} \simeq \frac{\alpha}{1-\alpha} .
$$

However, the hypothesis that the whole liquid is contributing to the vaporization process is a very strong one.

\subsection{Initial velocity $u_{0}=10 \mathrm{~m} / \mathrm{s}$}

A double rarefaction case is proposed for which the running fluid is liquid hydrogen in cryogenic conditions. Liquid hydrogen is initially at the pressure 2 bar and at the reference temperature $T_{r e f}=22.1 \mathrm{~K}$. A weak volume fraction of vapour $\alpha=0.01$ is initially added to the liquid. The initial discontinuity velocity is $u_{0}=10$ $\mathrm{m} / \mathrm{s}$.

The vapour pressure at the reference temperature is $P_{\text {vap }}\left(T_{\text {ref }}\right)=1.63$ bar. Parameters of the stiffened gas are given in Table 1 . The quantity $c_{\text {sinus }}$ is set to 3.15 $\mathrm{m} / \mathrm{s}$ following equation (21). The quantity $d P_{v a p} / d T$ is determined using the NIST thermodynamic table (NIST n.d.). Figure 3 presents the linear approximation of the vapour pressure on the temperature interval $[19,24] \mathrm{K}$. The value of $d P_{v a p} / d T$ is set to $38980 \mathrm{~Pa} / \mathrm{K}$. For all simulations, the mesh contains 5000 cells and the time step is set to $10^{-7} \mathrm{~s}$. Computations are performed using the first-order Rusanov scheme.

The pressure, the void fraction, the temperature and the velocity are plotted in Figure 4 , at time $\mathrm{t}=0.8 \mathrm{~ms}$, for both 4-equation models. The four expansion waves are clearly illustrated (two fast expansion waves and two slow evaporation fronts). The pressure drop under the initial value of the vapour pressure is around 0.2 bar. It is more pronounced using the 4-equation SG model and due to a larger cooling effect. The creation of void ratio is weak (around $20 \%$ ) and a little more void ratio is produced by the 4-equation sinus model. For the velocity profile, results obtained with both models are similar.

The vapour density $\rho_{v}$, the mass fraction of vapour $Y$, the B-factor and the speed of sound are plotted in Figure 5, at time $\mathrm{t}=0.8 \mathrm{~ms}$, for both 4-equation models. The mixture speed of sound of the sinus EOS is largely smaller than the SG relation, that can explain the greater quantity of vapour created by the sinus model, as observed for the mass fraction profile. Indeed, the mass transfer is linked to the quantity $1-c^{2} / c_{\text {wallis }}^{2}$ following equation (10). The B-factor is computed as the ratio between the actual temperature drop and the characteristic temperature drop $\Delta T^{*}$, following equation (34). Saturation values at the reference temperature $T_{\text {ref }}$ are used. For the 4-equation sinus, the maximal value is 0.375 to be compared with the ideal value given by the ratio $\alpha /(1-\alpha)=0.31$ evaluated with the maximal value of the void ratio ( $21 \%$ of error). For the 4-equation SG model, the maximal value is 0.49 to be compared with 0.22 ( $120 \%$ of error). Comparatively, the 4-equation sinus solution is in better agreement with the ideal value. The vapour density evolution is linked to the pressure and temperature profiles (it follows the stiffened gas EOS). As observed for $T$ and $P$, the 4-equation SG model provides a lower value in comparison with the 4-equation sinus model.

Finally, the effect of the numerical scheme is shown in Figure 6 where are plotted the pressure, the void ratio, the temperature and the velocity at time $t=0.8 \mathrm{~ms}$. For all variables, numerical solutions obtained with both schemes are similar. A small difference can be observed on the expansion fronts, which are stiffer using the 2ndorder Jameson scheme. In the following, only simulations performed with the Rusanov scheme will be presented.

\subsection{Initial velocity $u_{0}=100 \mathrm{~m} / \mathrm{s}$}

The same conditions are used except regarding the discontinuity velocity which is set to $u_{0}=100 \mathrm{~m} / \mathrm{s}$. Such high velocities are representative of flows occurring in turbopumps of rocket propulsion systems (see for example (Goncalves et al. 2010)). In this case, evaporation is much more intense resulting in a large cavitation pocket.

The estimation of the slope $d P_{v a p} / d T$ leads to difficulties due to the large temperature drop. A linear approximation on the temperature interval $[14,22] \mathrm{K}$ is presented in Figure 7 . The value of $d P_{v a p} / d T$ is around $24500 \mathrm{~Pa} / \mathrm{K}$. A parabolic approximation will be clearly better, but the model properties, especially the mixture speed of sound formulation, are more difficult to study. Secondly, using the linear approximation, we observe than the vapour pressure becomes negative if the temperature is smaller than $15 \mathrm{~K}$. This situation occurs with the initial discontinuity velocity $u_{0}=100 \mathrm{~m} / \mathrm{s}$. The pressure and the temperature are plotted in Figure 8, at time $\mathrm{t}=0.8 \mathrm{~ms}$, using the 4 -equation sinus model 
with $d P_{\text {vap }} / d T=24500 \mathrm{~Pa} / \mathrm{K}$. The cooling effect is important and the mixture temperature decreases to $14.9 \mathrm{~K}$. Negative values of the pressure appear in the middle of the tube. A similar result is obtained using the 4-equation SG model.

As a remedy, a smaller value is considered: $P_{\text {vap }} / d T=$ $20000 \mathrm{~Pa} / \mathrm{K}$. The pressure, the void fraction, the temperature and the vapour density are plotted in Figure 9 , at time $\mathrm{t}=0.8 \mathrm{~ms}$, for both 4 -equation models. Using the 4-equation SG model, negative values for the pressure and the vapour density are obtained. The temperature decreases up to $13.5 \mathrm{~K}$ that is lower than the triple point $(13.8 \mathrm{~K})$. On the other hand, using the 4-equation sinus model, the pressure and the vapour density remain positive. The temperature decreases to $14.3 \mathrm{~K}$. The pressure drop under the initial value of the vapour pressure is very strong, it reaches 1.5 bar. A large cavitation area is created for which the maximal value of the void ratio is $84 \%$.

Figure 12 presents the B-factor and the mixture speed of sound evolutions in the tube, at time $\mathrm{t}=0.8 \mathrm{~ms}$, computed with the 4-equation sinus model and with $d P_{\text {vap }} / d T=20000 \mathrm{~Pa} / \mathrm{K}$. The maximal value of the $\mathrm{B}$-factor is 5.5 to be compared with the ideal value estimated with the maximal void ratio (84\%) that is 5.25 ( $5 \%$ of error). The numerical simulation is in close agreement with the theoretical value. The large variations of the speed of sound are well illustrated: around $460 \mathrm{~m} / \mathrm{s}$ in the initial condition, $12 \mathrm{~m} / \mathrm{s}$ behind the expansion waves and $146 \mathrm{~m} / \mathrm{s}$ in the cavitation area. This non monotonous behaviour makes the numerical integration stiff.

Another strategy for the estimation of $d P_{v a p} / d T$ consists in splitting the linear approximation on two intervals, as plotted in Figure 10. The temperature at the intersection between the two lines is $18.15 \mathrm{~K}$. Values of $d P_{v a p} / d T$ are $9930 \mathrm{~Pa} / \mathrm{K}$ and $27260 \mathrm{~Pa} / \mathrm{K}$, respectively. The pressure, the void fraction, the temperature and the vapour density are plotted in Figure 11, at time $\mathrm{t}=0.8$ $\mathrm{ms}$, for both 4-equation models. Using the 4-equation SG model, as previously negative values are obtained for the pressure and vapour density. For both models, an irregularity is observed on profiles due to the change of slope $d P_{v a p} / d T$ around $18 \mathrm{~K}$. For the 4-equation sinus model, the temperature drop reaches $13.7 \mathrm{~K}$, which is under the triple point value.

\subsection{Shock-cavitation interaction, $u_{0}=100 \mathrm{~m} / \mathrm{s}$}

This case is similar to the previous one, except that the two ends of the tube are simultaneously closed once the flow starts. Therefore, a shock created at each end moves towards the center, resulting in shock-cavitation interaction and cavitation collapse. A uniform mesh of 5000 cells is used and the time step is set to $10^{-8} \mathrm{~s}$. Simulations are performed using the 4-equation sinus model with $d P_{\text {vap }} / d T=20000 \mathrm{~Pa} / \mathrm{K}$.

The pressure, the void ratio, the temperature and the vapour density are plotted at different times in Figure 13 (with a logarithmic scale for the pressure and the vapour density). The cavitation pocket grows up to time $t=$ $0.6 \mathrm{~ms}$. After this time, the shocks created at the ends meet the rarefaction waves generated at the center, and then interacts with the expanding cavitation interface. The cavitation collapse begins. The reduction of the cavitation area is clearly observed on the void ratio profile after time $t=0.6 \mathrm{~ms}$. From time $0.6 \mathrm{~ms}$ to $1.1 \mathrm{~ms}$, the cavitation pocket narrows but the maximal void ratio remains around $80 \%$. At time $t=1.2 \mathrm{~ms}$, the decrease of the void ratio is abrupt and the value in the middle of the tube is around $5 \%$.

The shock propagation through the rarefaction region is well illustrated on the pressure, the temperature and the vapour density profiles, up to time $t=0.6 \mathrm{~ms}$. Then shocks interacts with the expanding cavitation interface, resulting in a discontinuity forms at the interface. The cavitation collapse generates two shocks which propagate outwards. For the initial shocks, the maximum pressure is around 54 bar, whereas the maximal pressure for the two shocks created during the cavitation collapse is around 28 bar. At time $t=1.2 \mathrm{~ms}$ when the void ratio decreases abruptly, we observe a large increase of the vapour density up to $28 \mathrm{~kg} / \mathrm{m}^{3}$ and a warming effect about $6 \mathrm{~K}$ in comparison with the reference temperature. At time $t=1.3 \mathrm{~ms}$ (not presented), the temperature reaches $30 \mathrm{~K}$. The magnitude of both phenomena, the cooling effect due to the evaporation and the warming effect due to the collapse, is quite similar.

\section{Acknowledgments}

This paper is a revised and expanded version of a paper entitled Numerical Study of Cavitation in Liquid Hydrogen Flow presented at the $8^{\text {th }}$ International Conference on Thermal Engineering Theory and Applications - ICTEA 2015, Amman, Jordan, May 1821, 2015. Authors gratefully acknowledge support from the German Jordanian University and Ensma - Pprime.

\section{Conclusion}

The thermal effects in 1D cryogenic cavitating flows were studied. Two EOS were tested associated with a mass transfer closure based on the ratio between the mixture speed of sound and the Wallis speed of sound. Simulations were performed on inviscid rarefaction problems leading to a phase transition, for which the working fluid is liquid hydrogen at the temperature 22.1 K. Different initial velocities were considered and especially a high-speed case was investigated, corresponding to a realistic velocity in turbopump applications. Moreover, a shock-cavitation case was simulated leading to the collapse of the cavitation area. These cases lead to different concluding remark:

- As regard to the EOS comparison, the sinus model generated more vapour and a lower temperature 
depression than the SG model. For the high-speed case, the pressure and vapour density obtained with the SG model were negative, that constitutes a clear drawback.

- Problems appeared with the calibration of the parameter $d P_{v a p} / d T$ for the high-speed case because of the prediction of negative pressure. A piecewise approximation was tested and led to a temperature decrease lower than the triple point value. For this situation, a specific thermodynamic model have to be developped. Using a constant value $d P_{\text {vap }} / d T=20000 \mathrm{~Pa} / \mathrm{K}$, the simulated temperature drop was around $8 \mathrm{~K}$ and the result was in good agreement with the B-factor theory.

- For the shock-cavitation case, a warming effect was highlighted during the collapse for which the magnitude was quite similar to the cooling effect during the evaporation process.

Ongoing works are to pursue comparative analysis and to develop two-temperature models. A detailed thermal analysis under a range of physical conditions is planned in later phases of work to be reported in future publications.

\section{References}

Allaire, G., Clerc, S. \& Kokh, S. (2002), 'A fiveequation model for the simulation of interfaces between compressible fluids', Journal of Computational Physics 181(2), 577-616.

Ambroso, A., Chalons, C. \& Raviart, P.-A. (2012), 'A godunov-type method for the seven-equation model of compressible two-phase flow', Computers and Fluids 54, 6791.

Baer, M. \& Nunziato, J. (1986), 'A two-phase mixture theory for the deflagration-to-detonation transition (DDT) in reactive granular materials', Int. Journal of Multiphase Flow 12, 861-889.

Barberon, T. \& Helluy, P. (2005), 'Finite volume simulation of cavitating flows', Computers \& Fluids 34(7), 832-858.

Causon, D. \& Mingham, C. (2013), 'Finite volume simulation of unsteady shock-cavitation in compressible water', Int. Journal of Numerical Methods in Fluids 72, 632-649.

Chalons, C., Coquel, F., Kokh, S. \& Spillane, N. (2011), Large time-step numerical scheme for the seven-equation model of compressible two-phase flows, in 'Finite Volumes for Complex Applications VI Problems and Perspectives Springer Proceedings in Mathematics Volume', Vol. 4, pp. 225-233.

Charriere, B., Decaix, J. \& Goncalves, E. (2015), 'A comparative study of cavitation models in a venturi flow', European Journal of Mechanics - B/Fluids 49, 287-297.

Downar-Zapolski, P., Bilicki, Z., Bolle, L. \& Franco, J. (1996), 'The non-equilibrium relaxation model for one-dimensional flashing liquid flow', Int. Journal of Multiphase Flow 22(3), 473-483.
Florschuetz, L. \& Chao, B. (1965), 'On the mechanics of vapor bubble collapse', Journal of Heat Transfer 87, 209-220.

Franc, J.-P. \& Michel, J.-M. (2004), 'Fundamentals of cavitation', Springer.

Fujikawa, S., Okuda, M. \& Akamatsu, T. (1980), 'Nonequilibrium vapour condensation on a shock-tube endwall behind a reflected shock wave', Journal of Fluid Mechanics (183), 293-324.

Goncalves, E. (2013), 'Numerical study of expansion tube problems: Toward the simulation of cavitation', Computers \& Fluids 72, 1-19.

Goncalves, E. (2014), 'Modeling for non isothermal cavitation using 4-equation model', International Journal of Heat and Mass Transfer 76, 247-262.

Goncalves, E. \& Charriere, B. (2014), 'Modelling for isothermal cavitation with a four-equation model', International Journal of Multiphase Flow 59, 54-72.

Goncalves, E. \& Patella, R. F. (2009), 'Numerical simulation of cavitating flows with homogeneous models', Computers \& Fluids 38(9), 1682-1696.

Goncalves, E. \& Patella, R. F. (2010), 'Numerical study of cavitating flows with thermodynamic effect', Computers E Fluids 39(1), 99-113.

Goncalves, E. \& Patella, R. F. (2011), 'Constraints on equation of state for cavitating flows with thermodynamic effects', Applied Math. and Computation 217, 5095-5102.

Goncalves, E. \& Zeidan, D. (2015), 13) numerical study of cavitation in liquid hydrogen flow, in 'Proceedings of the 8th International Conference on Thermal Engineering Theory and Applications May 18-21, 2015, AmmanJordan, 2015'.

Goncalves, E., Patella, R. F., Rolland, J., Pouffary, B. \& Challier, G. (2010), 'Thermodynamic effect on a cavitating inducer in liquid hydrogen', Journal of Fluids Engineering 132(11), 111305.

Hsiao, C. \& Chahine, G. (2002), Prediction of vortex cavitation inception using coupled spherical and non-spherical models and unrans computations, in 'Proceedings of the $24^{\text {th }}$ Symposium on Naval Hydrodynamics'.

Hsiao, C., Jain, A. \& Chahine, G. (2006), Effect of gas diffusion on bubble entrainment and dynamics around a propeller, in 'Proceedings of the $26^{\text {th }}$ Symposium on Naval Hydrodynamics, Rome, Italy'.

Huang, B., Wu, Q. \& Wang, G. (2014), 'Numerical investigation of cavitating flow in liquid hydrogen', Int. Journal of Hydrogen Energy 39, 1698-1709.

Ishimoto, J. \& Kamijo, K. (2004), 'Numerical study of cavitating flow characteristics of liquid helium in a pipe', Int. Journal of Heat and Mass Transfer (47), 149-163.

Jameson, A., Schmidt, W. \& Turkel, E. (1981), Numerical solution of the Euler equations by finite volume methods using Runge-Kutta time stepping schemes, in 'AIAA Paper 81-1259'.

Kapila, A., Menikoff, R., Bdzil, J., Son, S. \& Stewart, D. (2001), 'Two-phase modeling of deflagration-todetonation transition in granular materials: reduced equations', Physics of fluids 13(10), 3002-3024. 
Kreeft, J. \& Koren, B. (2010), 'A new formulation of Kapila's five-equation model for compressible two-fluid flow, and its numerical treatment', Journal of Computational Physics 229(18), 6220-6242.

Metayer, O. L., Massoni, J. \& Saurel, R. (2004), 'Elaborating equations of state of a liquid and its vapor for two-phase flow models', Int. Journal of Thermal Sciences 43, 265276.

Moore, R. \& Ruggeri, R. (1968), Prediction of thermodynamic effects on developed cavitation based on liquid hydrogen and freon 114 data in scaled venturis, Technical report, NASA. TM D-4899.

NIST (n.d.), 'Webbook of chemistry', http://webbook.nist.gov/.

Pelanti, M. \& Shyue, K. (2014), 'A mixture-energy-consistent six-equation two-phase numerical model for fluids with interfaces, cavitation and evaporation waves', Journal of Computational Physics 259, 331-357.

Prosperetti, A. \& Plesset, M. (1978), 'Vapour-bubble growth in a superheated liquid', Journal of Fluid Mechanics 85, 349-368.

Rodio, M., Giorgi, M. D. \& Ficarella, A. (2012), 'Influence of convective heat transfer modeling on the estimation of thermal effects in cryogenic cavitating flows', Int. Journal of Heat and Mass Transfer 55, 6538-6554.

Rusanov, V. (1961), 'Calculation of interaction of non-steady shock waves with obstacles', Journal of Computational Mathematics and Physics 1, 267-279.

Saurel, R. \& Metayer, O. L. (2001), 'A multiphase model for compressible flows with interfaces, shocks, detonation waves and cavitation', Journal of Fluid Mechanics 431, 239-271.

Saurel, R., Petitpas, F. \& Abgrall, R. (2008), 'Modelling phase transition in metastable liquids: application to cavitating and flashing flows', Journal of Fluid Mechanics 607, 313-350.

Schmidt, D., Gopalakrishnan, S. \& Jasak, H. (2010), 'Multidimensional simulation of thermal non-equilibrium channel flow', International Journal of Multiphase Flow 36, 284-292.

Spina, G. L., de Mihieli Vitturi, M. \& Romenski, E. (2014), 'A compressible single-temperature conservative two-phase model with phase transitions', Int. Journal of Numerical Methods in Fluids 76, 282-311.

Stahl, H., Stepanoff, A. \& Phillipsburg, N. (1956), 'Thermodynamic aspects of cavitation in centrifugal pumps', Journal of Fluids Engineering 78, 1691-1693.

Tseng, C. \& Shyy, W. (2010), 'Modeling for isothermal and cryogenic cavitation', Int. Journal of Heat and Mass Transfer 53, 513-525.

Utturkar, Y., Wu, J., Wang, G. \& Shyy, W. (2005), 'Recent progress in modelling of cryogenic cavitation for liquid rocket propulsion', Progress in Aerospace Sciences 41, 558-608.

Wallis, G. (1967), 'One-dimensional two-phase flow', New York: McGraw-Hill.

Zeidan, D., Romenski, E., Slaouti, A. \& Toro, E. (2007), 'Numerical study of wave propagation in compressible two?phase flow', International Journal for Numerical Methods in Fluids 54(4), 393-417.
Zein, A., Hantke, M. \& Warnecke, G. (2010), 'Modeling phase transition for compressible two-phase flows applied to metastable liquids', Journal of Computational Physics 229(8), 2964-2998. 
Figure 1 The double rarefaction tube



Figure 2 Wave propagation diagram of the expansion tube

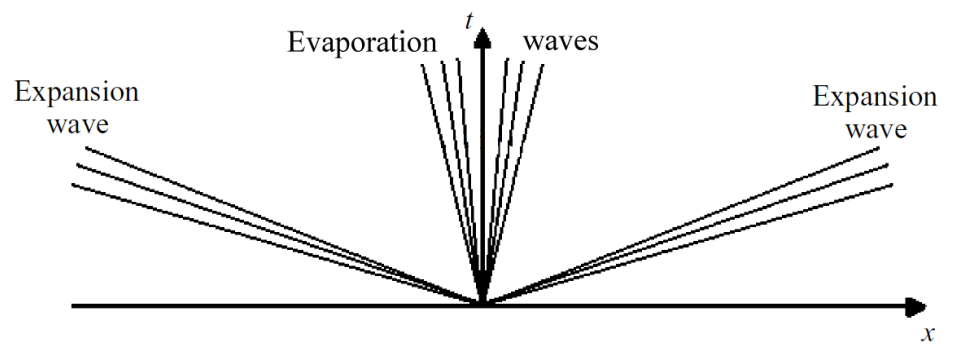

Figure 3 Variation of the vapour pressure with the temperature on the interval [19,24] K

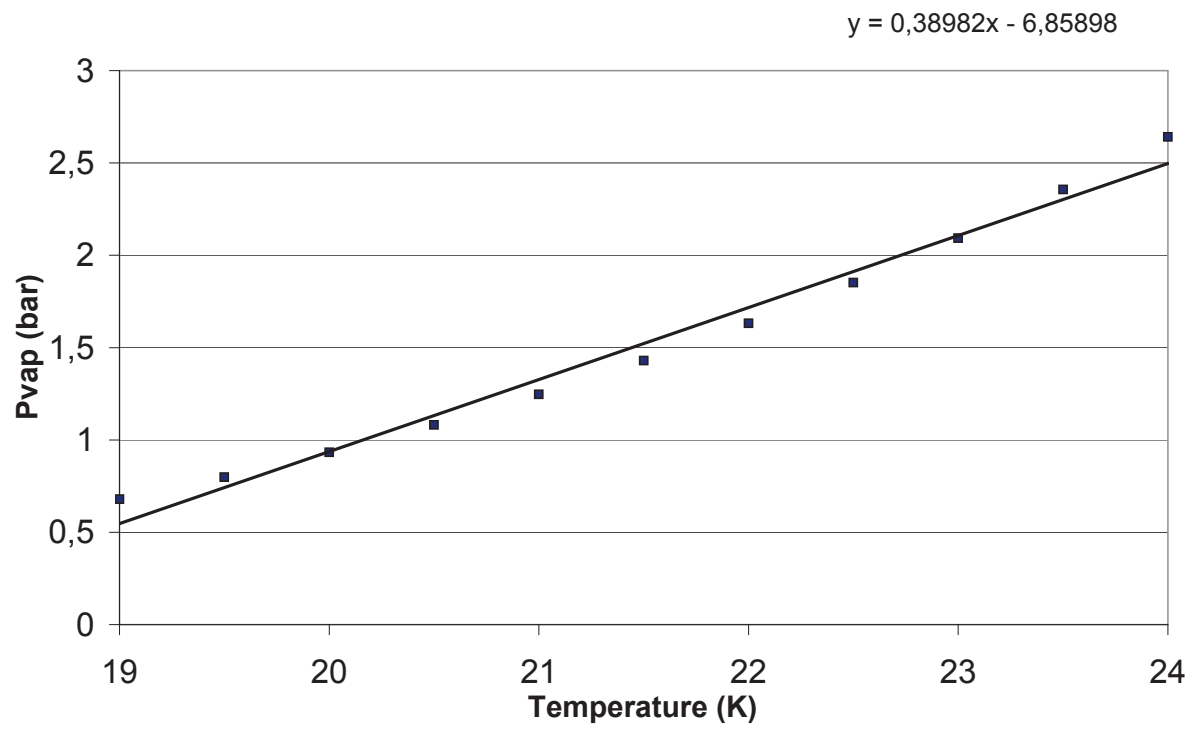


Figure 4 Double rarefaction with cavitation in $L H_{2}, u_{0}=10 \mathrm{~m} / \mathrm{s}, t=0.8 \mathrm{~ms}$. Models comparison. Pressure, void ratio, temperature and velocity
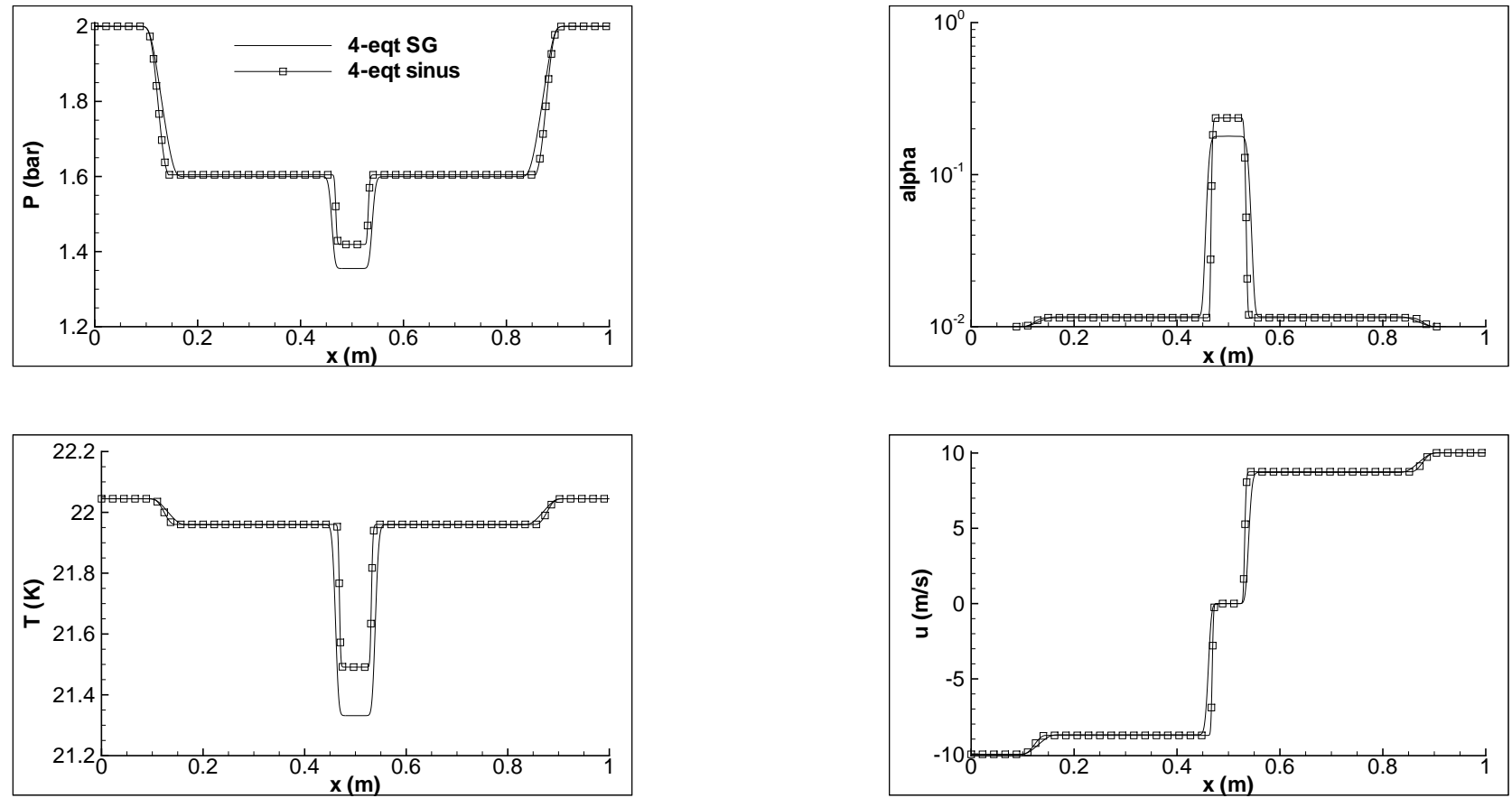

Figure 5 Double rarefaction with cavitation in $\mathrm{LH}_{2}, u_{0}=10 \mathrm{~m} / \mathrm{s}, t=0.8 \mathrm{~ms}$. Models comparison. Vapor density, mass fraction of vapour, B-factor and speed of sound
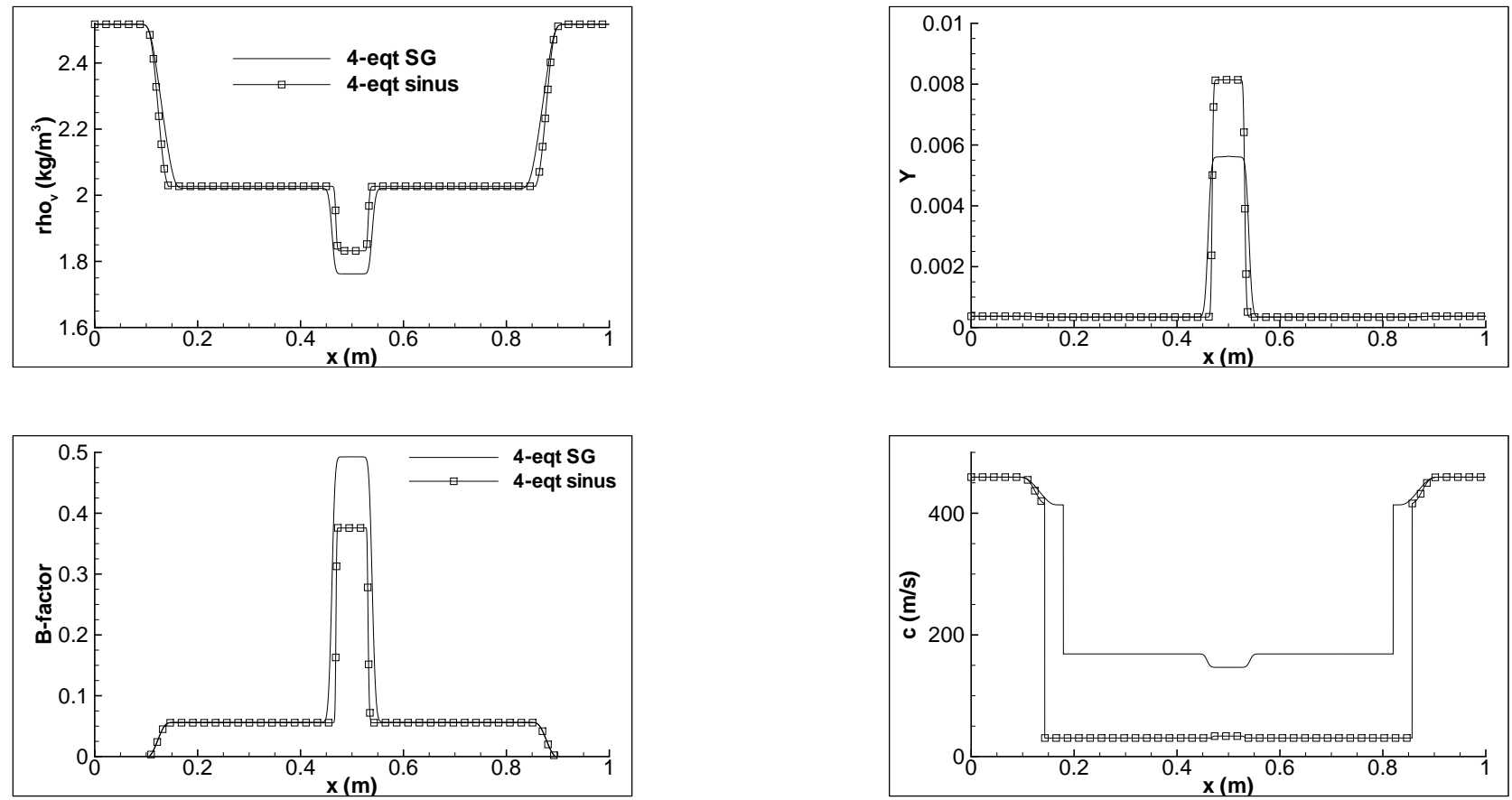
Figure 6 Double rarefaction with cavitation in $L H_{2}, u_{0}=10 \mathrm{~m} / \mathrm{s}, t=0.8 \mathrm{~ms}$. Numerical schemes comparison. Pressure, void ratio, temperature and velocity
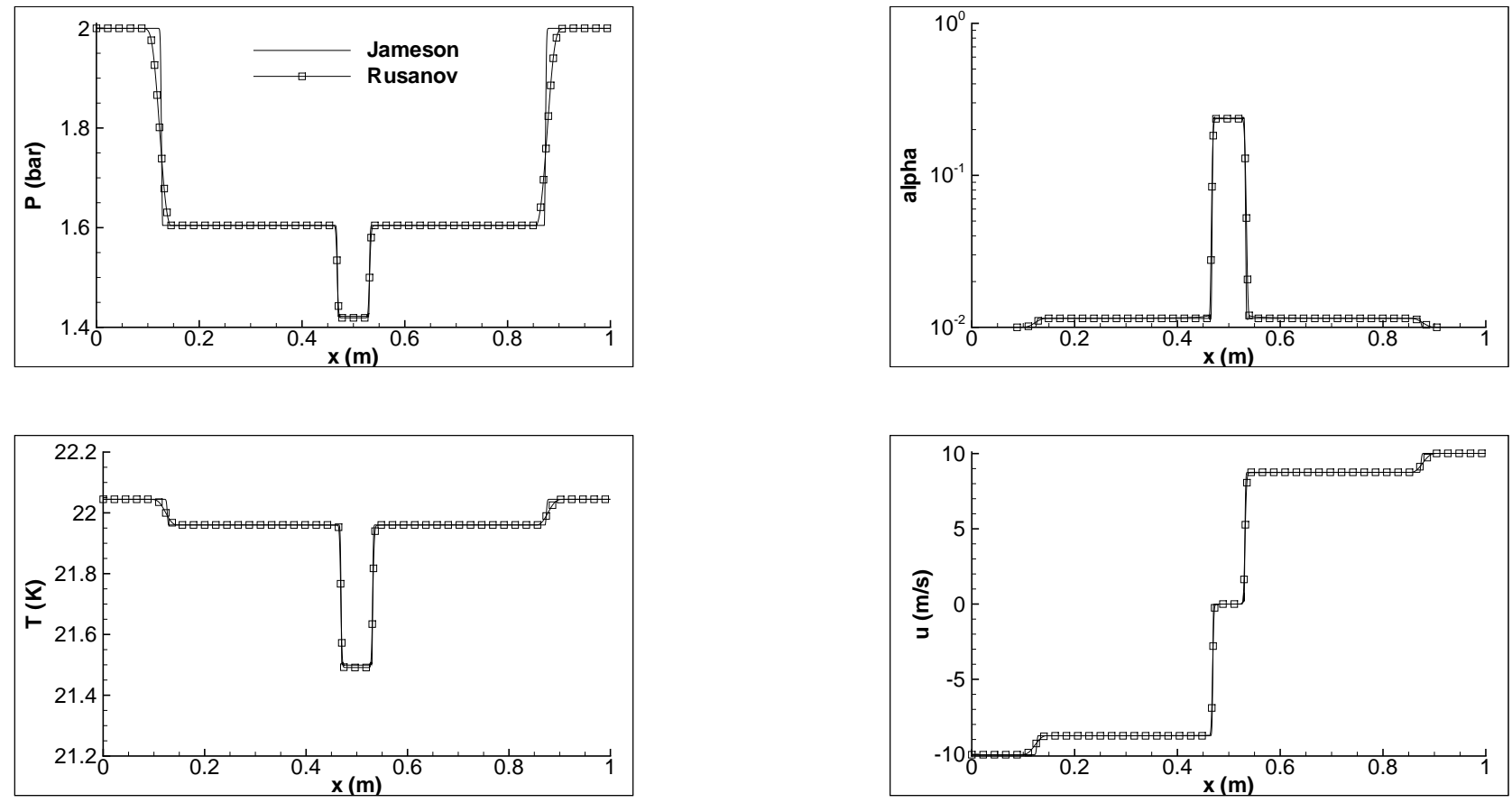

Figure 7 Variation of the vapour pressure with the temperature on the interval $[14,22] \mathrm{K}$

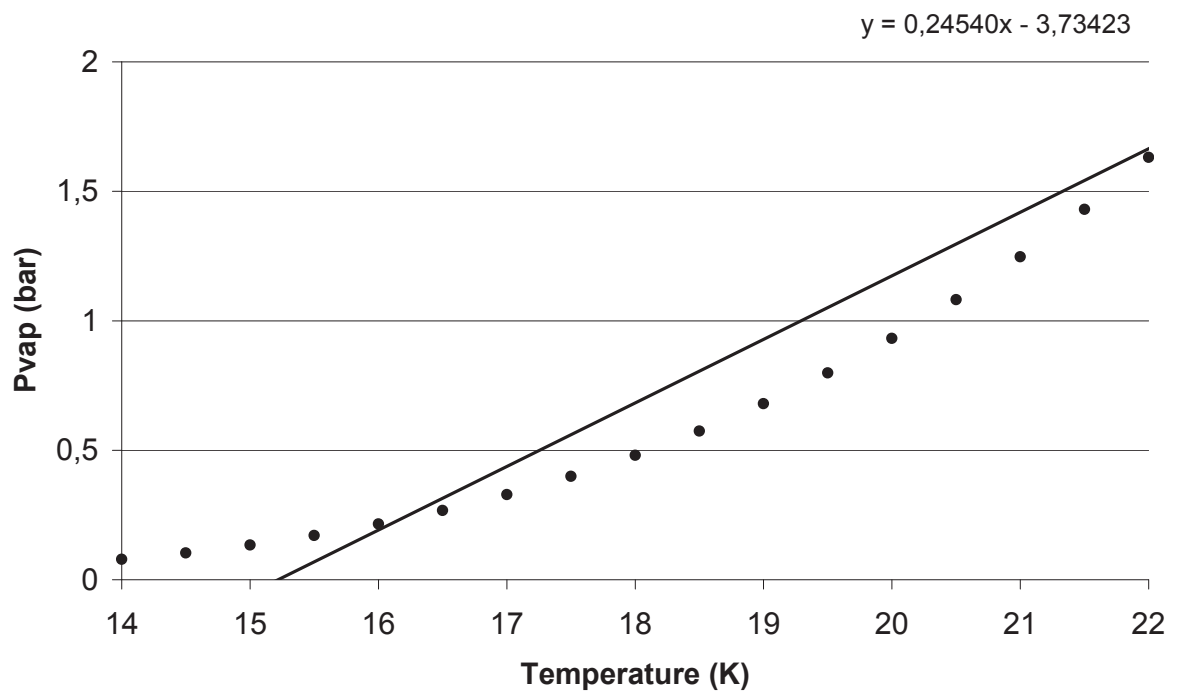


Figure 8 Double rarefaction with cavitation in $L H_{2}, u_{0}=100 \mathrm{~m} / \mathrm{s}, t=0.8 \mathrm{~ms}$. 4-equation sinus model, $d P_{v a p} / d T=24500$ $\mathrm{Pa} / \mathrm{K}$. Pressure and temperature
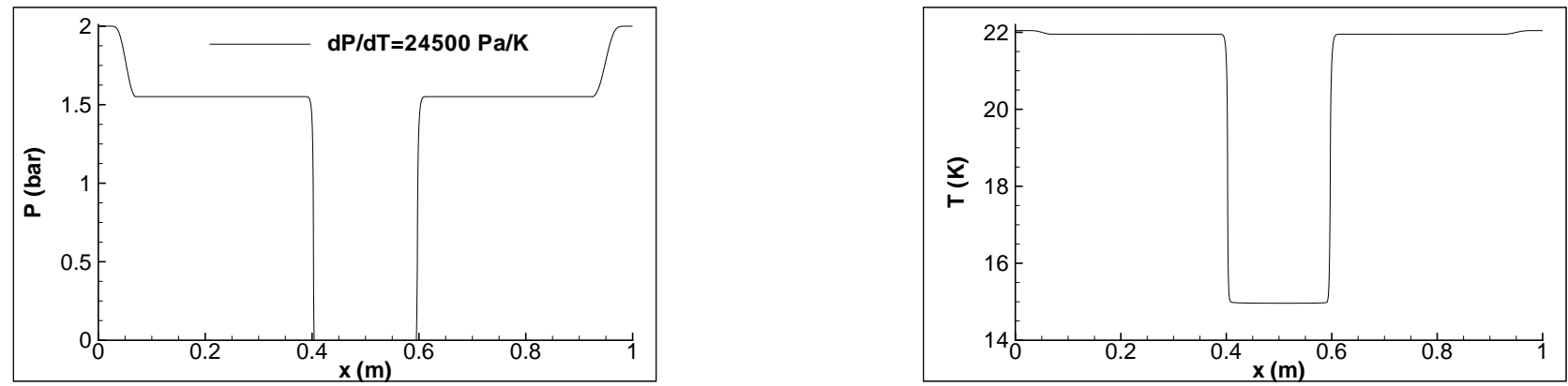

Figure 9 Double rarefaction with cavitation in $L H_{2}, u_{0}=100 \mathrm{~m} / \mathrm{s}, t=0.8 \mathrm{~ms}$. Models comparison, $d P_{v a p} / d T=20000$ $\mathrm{Pa} / \mathrm{K}$. Pressure, void ratio, temperature and vapour density


Figure 10 Double rarefaction with cavitation in $L H_{2}, u_{0}=100 \mathrm{~m} / \mathrm{s}, t=0.8 \mathrm{~ms}$. 4-equation sinus model. B-factor and speed of sound
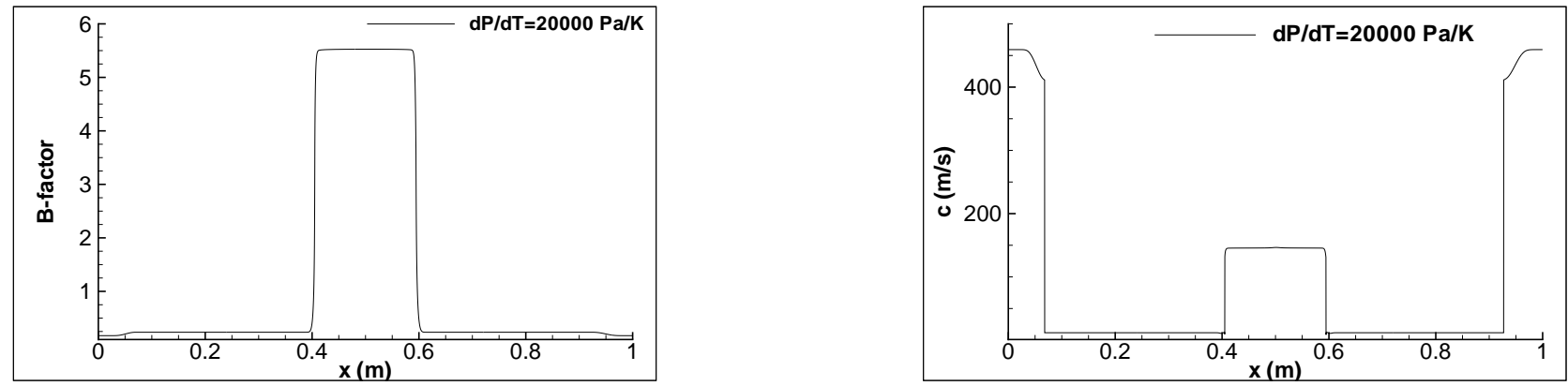
Figure 11 Double linear approximations of $P_{v a p}(T)$ on the interval $[14,22] \mathrm{K}$

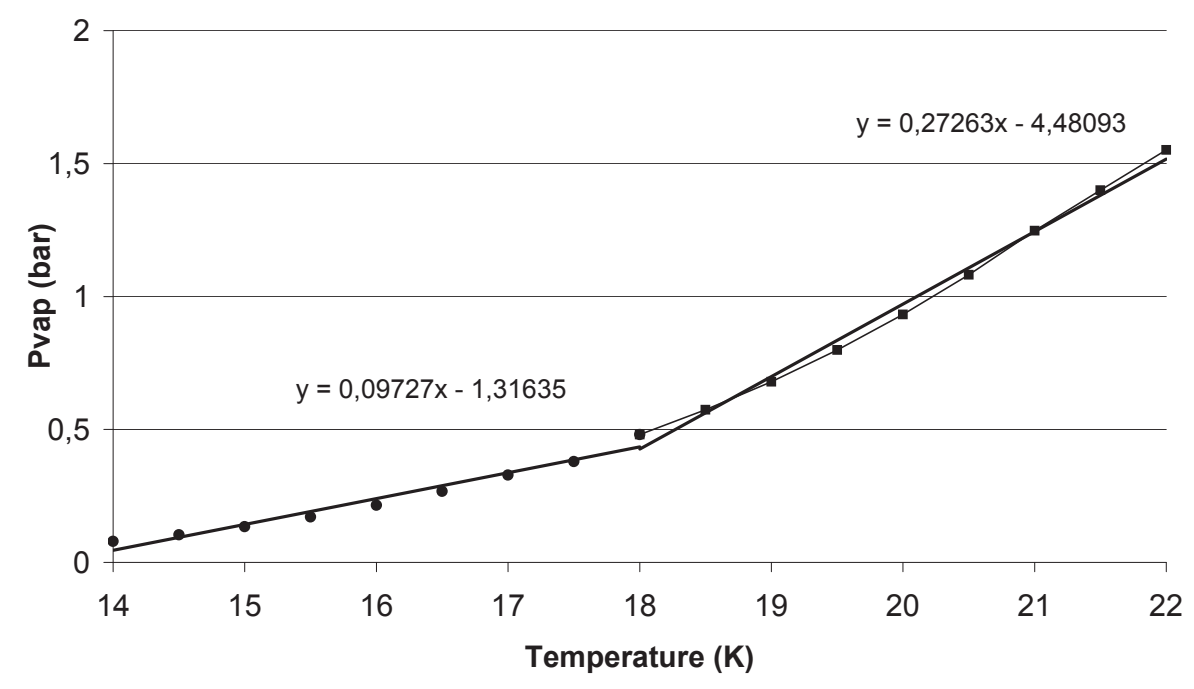

Figure 12 Double rarefaction with cavitation in $L H_{2}, u_{0}=100 \mathrm{~m} / \mathrm{s}, t=0.8 \mathrm{~ms}$. Models comparison, splitting of linear approximation of $d P_{v a p} / d T$. Pressure, void ratio, temperature and vapour density

Figure 13 Shock-cavitation interaction, $u_{0}=100 \mathrm{~m} / \mathrm{s}, t=1.2 \mathrm{~ms}$. 4-equation sinus model, $d P_{v a p} / d T=20000 \mathrm{~Pa} / \mathrm{K}$. Pressure, void ratio, temperature and vapour density

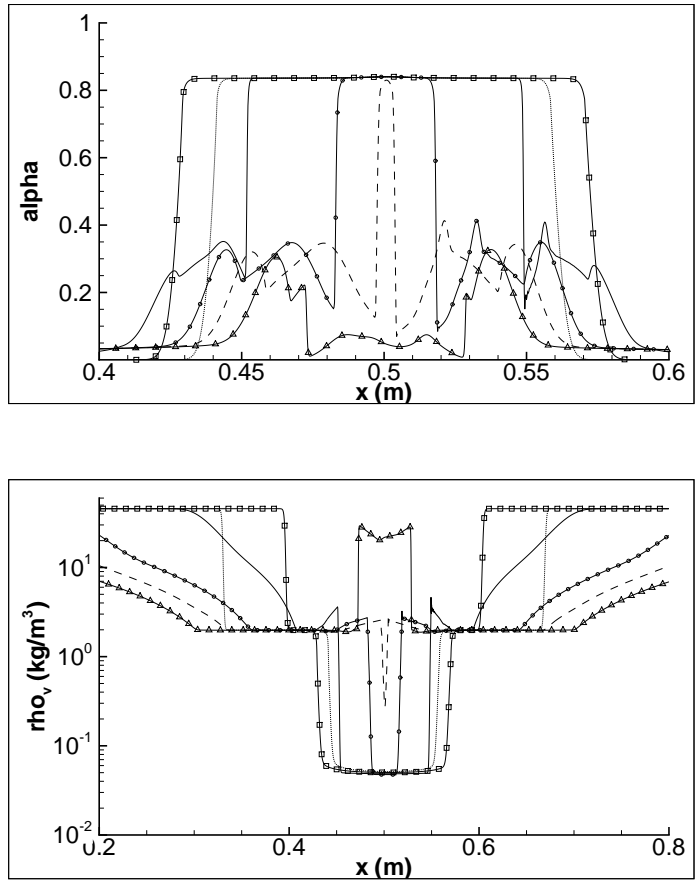